\section{Reducción del riesgo cardiovascular en mujeres adultas mediante ejercicio físico de sobrecarga}

\author{
RAFAEL ZAPATA-LAMANA ${ }^{1, a}$, IGOR CIGARROA ${ }^{2,3, b}$, \\ ERIK DÍAZ $^{4, \mathrm{c}}$, CARLOS SAAVEDRA $^{5, \mathrm{~d}}$
}

\section{Resistance exercise improves serum lipids in adult women}

\begin{abstract}
Background: Sedentariness is one of the main cardiovascular risk factors. Aerobic exercise can reduce the risk; however, resistance training seems to be more effective in reducing cardiovascular risk. Aim: To determine the metabolic effects of a 12 weeks resistance training program of high intensity and low volume in two groups of sedentary adult women. Material and methods: Forty women aged between 30 and 60 years were randomly assigned to an experimental group and a control group. Four kinds of strength exercises, using their own body weight were chosen. Plasma lipid profile and body composition were measured at baseline and at the end of the intervention. Results: A reduction in total cholesterol and triacylglycerol and an increase in $H D L$ cholesterol was observed in the experimental group after the training program. Conclusions: The high intensity and low volume training improved plasma lipid profile in this group of women.
\end{abstract}

(Rev Med Chile 2015; 143: 289-296)

Key words: Health Care; Physical fitness; Quality Indicators; Resistance training; Sedentary lifestyle.

\author{
'Escuela de Educación, Universidad \\ de Concepción, Los Ángeles, Chile. \\ ${ }^{2}$ Institut de Neurociències, Departa- \\ ment de Psiquiatria i Medicina Legal, \\ Universitat Autònoma de Barcelona, \\ Barcelona, España. \\ ${ }^{3}$ Carrera de Kinesiología, Facultad de \\ Salud, Universidad Santo Tomás, Los \\ Ángeles, Chile. \\ ${ }^{4}$ Centro de Investigación en Metabo- \\ lismo, Ejercicio y Salud (CIEMETS), \\ Departamento de Educación Física, \\ Universidad de la Frontera, Región \\ de la Araucanía, Chile. \\ ${ }^{5}$ Sociedad Chilena de Ciencias del \\ Ejercicio, Nutrición y Salud. \\ aprofesor de Educación Física, Mg. \\ en Medicina y Ciencias del Deporte, \\ Dr.@ Actividad Física y Deporte. \\ bLicenciado en Kinesiología, Mg. en \\ Neurorehabilitación, MSc. en Neuro- \\ ciencias y PhD@ en Neurociencias. \\ cNutricionista, MSc, PhD. \\ dProfesor de Educación Física, MSc. \\ Financiamiento: El autor Igor Ciga- \\ rroa declara haber recibido una beca \\ de perfeccionamiento conducente a \\ grado académico de la Universidad \\ Santo Tomás de Chile y apoyado \\ por CONYCYT/BECA CHILE/PAI \\ 72150035 durante el tiempo que \\ duró el estudio. \\ Recibido el 2 de septiembre de \\ 2014, aceptado el 26 de enero de \\ 2015. \\ Correspondencia a: \\ Rafael Zapata-Lamana \\ rafaelzapata@udec.cl
}

L a inactividad física se define como la ausencia de práctica regular de ejercicio físico, que disminuye progresivamente la condición física. Se ha asociado a una mayor prevalencia de enfermedades crónicas no trasmisibles como las enfermedades cardiovasculares, diabetes mellitus tipo $\mathrm{II}^{1-3} \mathrm{y}$ a factores de riesgo como el sobre peso, obesidad, la presión arterial y glicemia elevada 4 . Constituye el cuarto factor de riesgo más importante de mortalidad en todo el mundo ( $6 \%$ de defunciones a nivel mundial) y se estima que es la causante de $27 \%$ de la diabetes y cerca de $30 \%$ de las cardiopatías isquémicas ${ }^{5}$.

En relación a las orientaciones para la práctica de actividad física de la población, el Colegio Americano del Medicina del Deporte y la Asociación
Americana del Corazón ${ }^{6}$, plantean recomendaciones que indican acumular un mínimo de $150 \mathrm{~min}$ semanales de actividad física aeróbica moderada para población adulta (18 y 64 años). Paradójicamente, el departamento de salud y servicios humanos de los Estados Unidos de Norteamérica comunicó que para el 2010, 74\% de la población no sigue la simple recomendación de realizar al menos 30 min de ejercicio físico diario ${ }^{7}$.

Uno de los componentes principales en la génesis de las patologías crónicas modernas, metabólicas y cardiovasculares es la pérdida de la funcionalidad del tejido muscular derivada del sedentarismo que se caracteriza esencialmente por el compromiso en la capacidad de metabolización de grasas y glucosa ${ }^{8}$, así como el 
Ejercicio de sobrecarga disminuye el riesgo cardiovascular - R. Zapata-Lamana et al

aumento de la grasa intramuscular, en el hígado y páncreas.

Pese a que existen antecedentes en la población en general que indican que mejorando la condición física se optimizan a nivel celular los condicionantes de enfermedades crónicas ${ }^{4}$, transformándose así en una excelente herramienta terapéutica para combatir condiciones como la obesidad, sarcopenia y numerosas patologías provocadas por estilos de vida insanos ${ }^{10,11}$, los niveles de inactividad física son muy elevados, siendo mayor en mujeres (92,9\%) que en hombres $(88,6 \%)$ según la Encuesta Nacional de Salud $2010^{12}$. Dicha situación, se ve caracterizada con una sociedad que declara no tener tiempo libre para el desarrollo de ejercicio físico $(49,9 \%)$ de acuerdo a los datos presentados por la Encuesta Nacional de Hábitos de Actividad física y Deporte, realizada en Chile por el Instituto Nacional del Deporte (IND) ${ }^{13}$.

En la actualidad, se proponen nuevas modalidades de ejercicio con una metodología de intervalos de alta intensidad y corta duración que promueve diversos mecanismos de adaptación en el tejido muscular que, a su vez, desencadena en el incremento de la capacidad metabólica de este tejido y con ello una mejor homeostasis en el control de la glucosa y la oxidación de grasas ${ }^{14}$. En esta línea, el entrenamiento de sobrecarga de alta intensidad muscular y bajo volumen semanal de entrenamiento durante mucho tiempo ha sido aceptado como un medio para mantener y desarrollar fuerza y resistencia muscular, sin embargo, su relación beneficiosa con factores asociados a la salud y la enfermedad crónica han sido recientemente reconocidas en la literatura científica ${ }^{15}$.

El plan de entrenamiento propuesto es una alternativa terapéutica a las actuales recomendaciones tradicionales de actividad física proporcionadas por diversas instituciones internacionales como el Colegio Americano de Medicina del Deporte y la Asociación Americana del Corazón ${ }^{6}$.

El presente estudio tuvo como objetivo evaluar los efectos de un programa de ejercicio físico de sobrecarga de alta intensidad y bajo volumen en un grupo de mujeres adultas con sobrepeso, en comparación con otro grupo de mujeres adultas con sobrepeso que realizaban sólo sus actividades de la vida diaria sin la inclusión de un programa de ejercicio.

\section{Pacientes y Método}

Diseño

Estudio cuantitativo, de diseño experimental con grupo control, con una muestra no probabilística, por conveniencia.

\section{Muestra}

Cuarenta mujeres sedentarias trabajadoras de la Universidad de Concepción (UdeC) Campus Los Ángeles fueron divididas en dos grupos; uno experimental (GE; $\mathrm{n}=20$; edad (años): 44,3 \pm 2,1; estatura $(\mathrm{cm}): 157,1 \pm 1,3$ y peso $(\mathrm{kg}): 73,9 \pm$ $2,4)$ ) que realizó un programa de ejercicio físico de sobrecarga de alta intensidad y bajo volumen (ESAIBV) adicionado a las actividades de la vida diaria $\mathrm{y}$, por otro lado, un grupo control $(\mathrm{GC} ; \mathrm{n}=20$; edad (años): 45,9 $\pm 2,6$; estatura (cm): 154,8 $\pm 1,1$ y peso $(\mathrm{kg}): 69,2 \pm 2,2)$ ) que realizaban sólo sus actividades de la vida diaria sin la inclusión de un programa de ejercicio. Todas las mujeres fueron asignadas aleatoriamente a un grupo. Luego, los grupos fueron sometidos a una evaluación metabólica inicial (EMI), compuesta de evaluación complementaria, composición corporal y perfil lipídico. Se realizó en este mismo orden y 4 días previos al inicio del programa ESAIBV.

El grupo experimental sometido al programa ESAIBV entrenó durante 24 sesiones, 2 veces por semana durante un período de 3 meses. Las sesiones de ejercicio tenían una duración de 36 min aproximadamente y se realizaron entre las 11:00 y la 13:00 h o entre las 17:00 y 19:00 h en el Laboratorio de Ciencias del Trabajo de la UdeC, con condiciones estándar de humedad $(50 \% \pm 10)$ y temperatura $\left(21^{\circ} \mathrm{C} \pm 2\right)$. Al terminar el programa ESAIBV, fueron eliminadas 2 mujeres del GE por no asistir a un mínimo de $80 \%$ de sesiones de entrenamiento y 5 mujeres del GC por no realizar la EMI. Así, la muestra quedo en 33 mujeres; 18 en el GE y 15 en el GC.

\section{Procedimientos}

\section{Evaluación metabólica inicial}

Antropometría: Se calculó índice de masa corporal (IMC), mediante la fórmula de peso/ $\left(\text { estatura }^{2}\right)^{16}$. Se evaluaron estatura y peso mediante un balanza tallímetro marca Tanita modelo WB-3000, con sensibilidad de $0,1 \mathrm{~cm}$ y $0,1 \mathrm{~kg}$ respectivamente. 
Composición corporal: Se evaluó el porcentaje de grasa (\%) y masa libre de grasa. Para ello, se realizó una medición pie-pie mediante 4 electrodos (metatarso-calcáneo), a través de bioimpedianciometría, en equipo marca Tanita modelo SC-331 con una sensibilidad de $1 \%$ para porcentaje de grasa y $0,1 \mathrm{~kg}$ para masa libre de grasa ${ }^{17}$.

Perfil lipídico: (triglicéridos, colesterol total, HDL, LDL, VLDL y relación colesterol total/ HDL). Para determinar el perfil lipídico se extrajeron muestras de sangre venosa tras $12 \mathrm{~h}$ de ayuno. Los resultados fueron obtenidos mediante la extracción de $4 \mathrm{ml}$ de sangre venosa y analizados empleando tecnología Roche ${ }^{18}$. Todos estos análisis se realizaron en dependencias del Laboratorio Clínico Central de la ciudad de Los Ángeles, certificado con Norma de Calidad ISO 15189.

\section{Programa de ejercicio de sobrecarga de alta intensidad y bajo volumen}

El programa de ESAIBV corresponde al trabajo de segmentos musculares aislados y en distintas posiciones de realización. En cada ejercicio, los sujetos realizaron el máximo número de repeticiones durante $1 \mathrm{~min}$ (utilizando como resistencia la porción involucrada del peso corporal intentando llegar a la fatiga de los grupos musculares en ejercicio, seguido de dos minutos de descanso y repetida esta acción en tres ocasiones. La secuencia temporal en el uso de los sustratos energéticos durante el trabajo muscular anaeróbico es suficiente como para inducir aumentos de la temperatura, $\mathrm{pH}$ e impactar los depósitos de glicógeno específicos de los músculos que participan en la contracción muscular e incidir en la síntesis de proteínas y en la biogénesis mitocondrial ${ }^{14,19,20}$. Previo a la aplicación de los ESAIBV fue necesario evaluar la carga (peso) que cada segmento corporal era capaz de soportar hasta llegar a la fatiga en un período aproximado de 1 min.

Los ejercicios seleccionados fueron: Flexión de brazo, flexión de tronco, extensión de brazo y extensión de pierna, con 3 series de trabajo por cada ejercicio, sumado a los intervalos de pausa, el tiempo total de cada sesión fue de 36 min mientras que el tiempo efectivo de trabajo fue de 12 min. Después de 3 días de acabado el programa de ejercicio, se realizó una evaluación metabólica final (EMF). Para determinar edad, antecedentes de tratamiento farmacológico, historia de enfermedades señaladas en los criterios de inclusión y exclusión, se completaron fichas de registro para cada participante.

El protocolo fue aprobado por el Comité de ética de la UdeC. Las entrevistas y mediciones clínicas se llevaron a cabo previa firma de cartas de consentimiento informado, respetando la autonomía del sujeto según la ley 20.584. Todos los procedimientos utilizados para la realización del presente estudio han seguido los principios éticos para las investigaciones médicas en seres humanos según se recoge en la Declaración de Helsinki.

\section{Análisis estadísticos}

Todos los resultados fueron expresados como promedio \pm errores estándar de la media. Los datos se analizaron utilizando el programa SPSS (v.19.0, SPSS Inc., USA). Para determinar normalidad de los datos dependientes se utilizó el test de Shapiro-Wilk y para determinar la homogeneidad de varianzas la prueba de Levene. Se usó una prueba $\mathrm{T}$ de muestras independientes para determinar homogeneidad entre los grupos al inicio del experimento. Para determinar el efecto del grupo y su interacción en las variables en estudio se utilizó análisis de varianza de medidas repetidas. Se hizo una prueba $\mathrm{T}$ de muestras relacionadas para determinar diferencias entre evaluación inicial y final. Un margen de error alfa de $5 \%(\mathrm{p}<0,05)$ se usó en todas las comparaciones.

\section{Resultados}

Todas las variables se distribuyeron en forma normal bajo la prueba de Shapiro Wilk y mostraron homogeneidad de varianzas con la prueba de Levene, lo que permitió hacer estadística paramétrica. Luego, con una prueba $\mathrm{T}$ de muestras independientes se observó que todas las variables evaluadas no tenían diferencias de medias entre grupos, lo que indicó que ambos grupos eran homogéneos y se encontraban en igualdad de condiciones antes de comenzar con la intervención.

\section{Antropometría}

Se observó una interacción intra-sujeto entre el programa ESAIBV y las evaluaciones inicialfinal. Por un lado, el GE bajó sus promedios de IMC y por otro el GC los subió $(\mathrm{F}(1,31)=10,103$, $\mathrm{p}=0,003)$. Además, al comparar con la evaluación inicial, el GC mostró un aumento significativo de su IMC $(p=0,005)($ Tabla 1$)$. 


\section{Tabla 1. Variables antropométricas, composición corporal, perfil lipídico y clasificación} de colesterol total

\begin{tabular}{|c|c|c|}
\hline Variables & Grupo experimental & Grupo control \\
\hline \multicolumn{3}{|c|}{$\begin{array}{l}\text { Antropometría } \\
\text { Índice de masa corporal IMC (kg/m²) }\end{array}$} \\
\hline Evaluación inicial & $29,9 \pm 0,89[28,06-31,83]$ & $28,9 \pm 1,03[26,72-31,12]$ \\
\hline Evaluación final & $29,8 \pm 0,94[27,82-31,78]$ & $29,4 \pm 1,09[27,08-31,78]$ \\
\hline$\Delta \%$ & $0,1(\downarrow 0,33 \%)$ & $0,5(\uparrow 1,73 \%)$ \\
\hline$p=$ & 0,314 & $0,005^{*}$ \\
\hline \multicolumn{3}{|c|}{$\begin{array}{l}\text { Composición corporal } \\
\text { Porcentaje de grasa corporal (\%) }\end{array}$} \\
\hline Evaluación inicial & $39,1 \pm 1,15[36,74-41,57]$ & $37,5 \pm 0,89[35,63-39,43]$ \\
\hline Evaluación final & $39,1 \pm 1,23[36,53-41,72]$ & $38,1 \pm 0,88[36,22-39,98]$ \\
\hline$\Delta \%$ & $0(0 \%)$ & $0,6(\uparrow 1,6 \%)$ \\
\hline$p=$ & 0,958 & 0,079 \\
\hline \multicolumn{3}{|l|}{ Masa libre de grasa } \\
\hline Evaluación inicial & $44,5 \pm 0,74[42,96-46,10]$ & $43,3 \pm 0,82[41,56-45,08]$ \\
\hline Evaluación final & $44,8 \pm 0,83[43,06-46,54]$ & $43,7 \pm 0,95[41,61-45,70]$ \\
\hline$\Delta \%$ & $0,3(\uparrow 0,67 \%)$ & $0,4(\uparrow 0,92 \%)$ \\
\hline$p=$ & 0,535 & 0,606 \\
\hline \multicolumn{3}{|l|}{ Perfil lipídico } \\
\hline Evaluación inicial & $202,6 \pm 8,03[185,62-219,49]$ & $195,9 \pm 9,85[174,75-216,98]$ \\
\hline Evaluación final & $186,1 \pm 6,41[172,59-199,63]$ & $207,9 \pm 8,82[188,96-226,77]$ \\
\hline$\Delta \%$ & $16,5(\downarrow 8,14 \%)$ & $12(\uparrow 6,13 \%)$ \\
\hline$p=$ & $0,005^{*}$ & 0,001 * \\
\hline \multicolumn{3}{|l|}{ Triglicéridos (mg/dl) } \\
\hline Evaluación inicial & $119,9 \pm 9,95[98,94-140,95]$ & $135,9 \pm 11,03[112,28-159,59]$ \\
\hline Evaluación final & $102,8 \pm 9,21[83,40-122,27]$ & $139,8 \pm 11,22[115,74-163,86]$ \\
\hline$\Delta \%$ & $17,1(\downarrow 14,26 \%)$ & $3,9(\uparrow 2,87 \%)$ \\
\hline$p=$ & $0,006^{*}$ & 0,430 \\
\hline \multicolumn{3}{|c|}{ Colesterol LDL (mg/dl) } \\
\hline Evaluación inicial & $122,0 \pm 7,77[105,61-138,40]$ & $110,1 \pm 9,13[90,49-129,64]$ \\
\hline Evaluación final & $106,2 \pm 6,10[93,35-119,09]$ & $115,7 \pm 9,90[94,45-136,89]$ \\
\hline$\Delta \%$ & $15,8(\downarrow 12,95 \%)$ & $5,6(\uparrow 5,09 \%)$ \\
\hline$p=$ & $0,001 *$ & $0,034^{*}$ \\
\hline \multicolumn{3}{|c|}{ Colesterol HDL (mg/dl) } \\
\hline Evaluación inicial & $59,5 \pm 4,32[50,39-68,61]$ & $59,3 \pm 3,87[51,4-67,63]$ \\
\hline Evaluación final & $64,2 \pm 3,98[55,78-72,55]$ & $52,1 \pm 3,29[45,08-59,19]$ \\
\hline$\Delta \%$ & $4,7(\uparrow 7,90 \%)$ & $7,2(\downarrow 12,14 \%)$ \\
\hline$p=$ & 0,172 & $0,013^{*}$ \\
\hline \multicolumn{3}{|c|}{ Colesterol VLDL (mg/dl) } \\
\hline Evaluación inicial & $25,6 \pm 2,09[21,15-29,98]$ & $28,0 \pm 2,16[23,38-32,62]$ \\
\hline Evaluación final & $20,7 \pm 1,75[16,97-24,37]$ & $28,8 \pm 2,71[23,00-34,60]$ \\
\hline$\Delta \%$ & $4,9(\downarrow 19,14 \%)$ & $0,8(\uparrow 2,86 \%)$ \\
\hline$p=$ & $0,001 *$ & 0,609 \\
\hline \multicolumn{3}{|c|}{ Clasificación de colesterol total (mg/dl) < 200/200 a 240/ > 240} \\
\hline Evaluación inicial & $6 / 10 / 2$ & $7 / 7 / 1$ \\
\hline Evaluación final & $12 / 6 / 0$ & $5 / 8 / 2$ \\
\hline
\end{tabular}

Los resultados son presentados en promedio \pm errores estándar de la media e [intervalo de confianza], para el perfil lípido los datos son medidos en miligramos/decilitros (Mg/dl). GE: grupo experimental, $\mathrm{n}: 15$ y GC: grupo control, n: 18. $\Delta \%$ : magnitud de cambio entre la evaluación inicial y final, * $p<0,05$ para pruebas T entre evaluación inicial y evaluación final. 


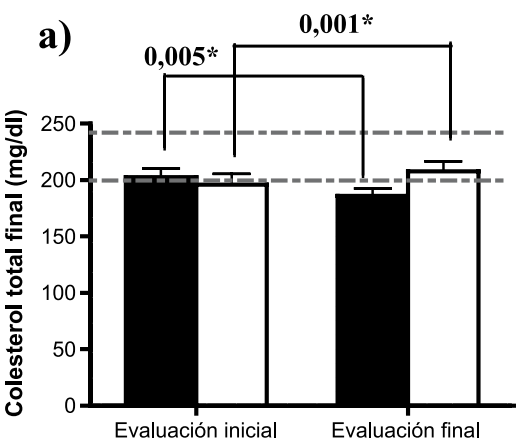

b)

Grupo experimental

$\square$ Grupo control
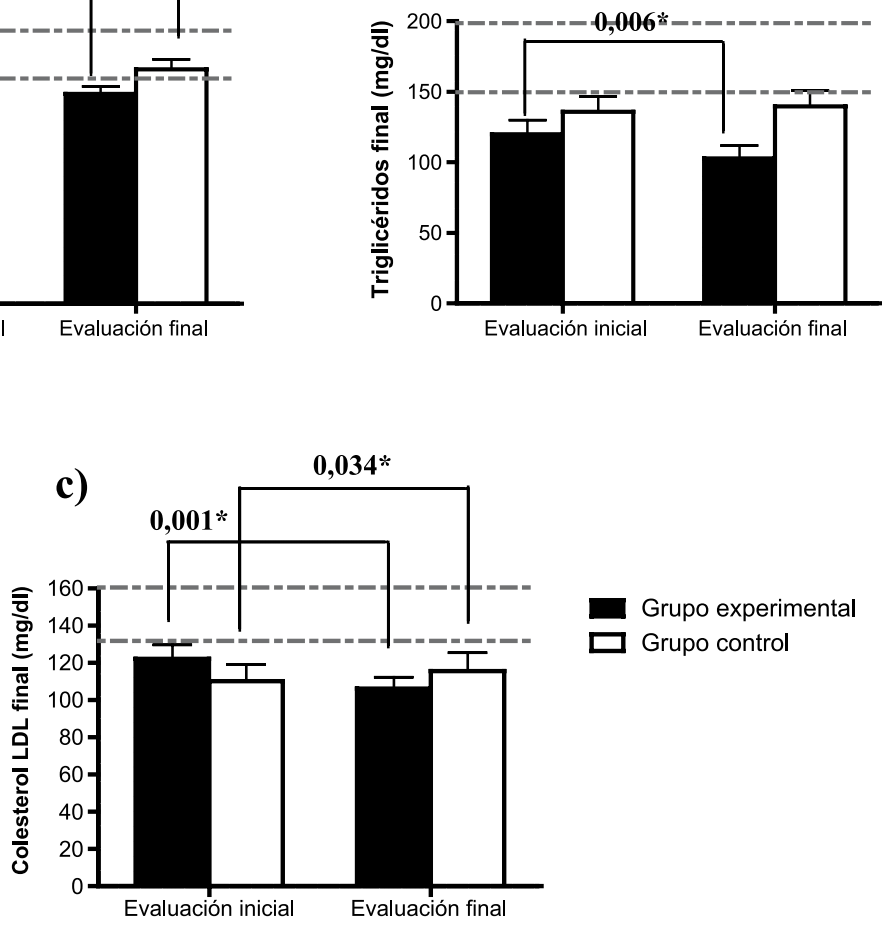

d)

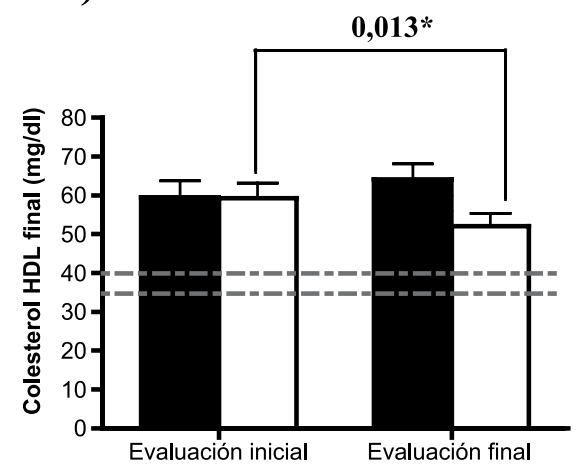

e)

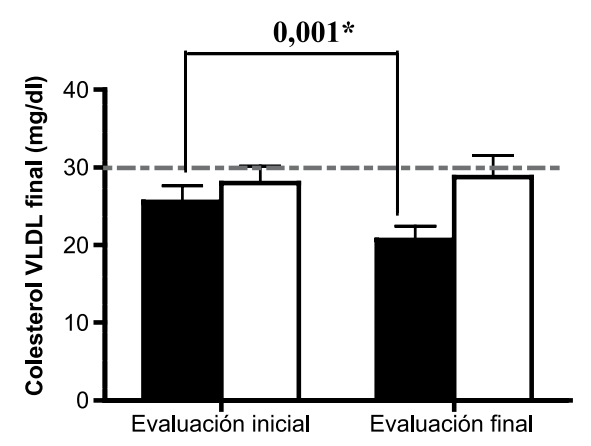

Grupo experimental Grupo control

Figura 1. Perfil lipídico. a) Colesterol total (colesterol total medido en miligramos/decilitros (mg/dl)) riesgo Mínimo: menor que $200 \mathrm{mg} / \mathrm{dl}$ y riesgo alto: mayor a $240 \mathrm{mg} / \mathrm{dl}$ en evaluación inicial y evaluación final de ambos grupos; b) Triglicéridos (triglicéridos medidos en $(\mathrm{mg} / \mathrm{dl})$ ) riesgo mínimo: menor de $150 \mathrm{mg} / \mathrm{dl}$ y riesgo alto mayor: $200 \mathrm{mg} / \mathrm{dl}$ en evaluación inicial y evaluación final de ambos grupos; c) Colesterol LDL (colesterol LDL medido en (mg/dl)) riesgo mínimo: menor a $130 \mathrm{mg} / \mathrm{dl}$ y riesgo mínimo: mayor a 160 mg/dl en evaluación inicial y evaluación final de ambos grupos; d) Colesterol HDL (colesterol HDL medido en $\mathrm{mg} / \mathrm{dl}$ ) riesgo mínimo: mayor a $40 \mathrm{mg} / \mathrm{dl}$ y riesgo alto: menor a $35 \mathrm{mg} / \mathrm{dl}$ en evaluación inicial y evaluación final de ambos grupos; e) Colesterol VLDL (colesterol VLDL medido en (mg/dl)) hasta $30 \mathrm{mg} / \mathrm{dl}$ en evaluación inicial y evaluación final de ambos grupos. $\mathrm{p}<0,05$ respecto a la evaluación inicial dentro del grupo. 
Ejercicio de sobrecarga disminuye el riesgo cardiovascular - R. Zapata-Lamana et al

\section{Composición corporal}

No hubo diferencias estadísticas en las variables porcentaje de grasa y masa libre de grasa $(\mathrm{kg})$, tanto en la comparación entre los grupos como entre la evaluación inicial vs evaluación final dentro del grupo. Destaca que ambos grupos presentan un exceso del porcentaje de grasa corporal, superando $30 \%$ en ambos casos (Tabla 1 ).

\section{Perfil lipídico}

No se encontraron diferencias significativas en la comparación de ambos grupos. Pese a ello, se observó una interacción intra-sujeto entre el programa de ejercicio y las evaluaciones inicialfinal. Por un lado, el GE bajó significativamente los niveles de colesterol total $((\mathrm{F}(1,31)=20,740$; $\mathrm{p}=0,000)$, de triglicéridos $(\mathrm{F}(1,31)=8,034$; $\mathrm{p}=0,008)$, de colesterol LDL $(\mathrm{F}(1,31)=17,978$; $\mathrm{p}=0,000)$, de colesterol VLDL $(\mathrm{F}(1,31)=8,425$; $\mathrm{p}=0,007)$ y subió los niveles de colesterol HDL $(\mathrm{F}(1,31)=7,737 ; \mathrm{p}=0,009)$ en comparación a su evaluación inicial. Por otro lado, el GC hizo todo lo contrario. Lo que determinó que al comparar los grupos por separado, el grupo que realizó el programa ESAIBV bajara $8,14 \%$ sus niveles de colesterol total, $14,26 \%$ sus niveles de triglicéridos, $12,95 \%$ sus niveles de colesterol LDL, 19,14\% sus niveles de colesterol VLDL y aumentara 7,9\% sus niveles de colesterol HDL, por el contrario, el GC aumentó $6,13 \%$ sus niveles de colesterol total, $2,87 \%$ sus niveles de triglicéridos, 5,09\% sus niveles de colesterol LDL, 2,86\% sus niveles de colesterol VLDL y disminuyó $12,14 \%$ sus niveles de colesterol HDL (Figura 1 y Tabla 1). La Tabla 1 muestra que 6 sujetos $(33,33 \%)$ del GE salieron de la condición de riesgo y lograron normalizar el colesterol total. Situación contrario sucedió con el GC que aumentó la cantidad de sujetos en esta condición de riesgo de 8 a 10 (53,33\% al 66,67\%).

\section{Discusión}

En el presente estudio se pudo observar que el programa ESAIBV fue capaz de mejorar la salud cardiovascular de mujeres sedentarias en comparación a su evaluación inicial. Modificando significativamente los lípidos sanguíneos y el colesterol. Se logró una importante tasa de recuperación en las personas que tuvieron inicialmente el perfil lipídico alterado, lo que podría implicar una potencial capacidad terapéutica.
En relación a la composición corporal, el programa ESAIBV no fue capaz de disminuir el IMC en el grupo que lo practicó, estos resultados podrían deberse a una dosis insuficiente de ejercicio físico, determinada por la duración y la frecuencia semanal del mismo. Además, no se efectuó control sobre la ingesta dietética, no pudiendo descartar el efecto que pudo haber tenido en la ausencia o mayores resultados. Es común que las personas tiendan a incrementar su ingesta al realizar ejercicio físico asumiendo que al tener mayor gasto tienen mayores necesidades energéticas.

Nuestros resultados son coincidentes a los publicados por Álvarez y col. ${ }^{18}$, donde se demuestra también que 2 sesiones semanales por 3 meses de duración con un entrenamiento de similares características no lograron impactar significativamente la composición corporal en su grupo de estudio. Coincidente con Balducci y col. ${ }^{21}$, podemos afirmar que no es necesario inducir cambios en el peso y composición corporal, para lograr modificar positivamente el perfil lipídico de los sujetos. Esto implica que la velocidad de cambio de estas variables pudiera ser mucho más rápida de lo esperado.

Coincidente con las mejoras en el perfil lipídico, de los estudios de Haskell ${ }^{22}$, Boreham y col. ${ }^{23}$, Balducci y col. ${ }^{21}$, Álvarez y col. ${ }^{24}$, que utilizando similares programas de intervención se demostró que el grupo experimental logró disminuir los triglicéridos y VDLD y aumentar los HDL en comparación al grupo control. Estos resultados concuerdan también con los de Babraj y col. ${ }^{19}$, donde se demuestra que la implementación de un programa de ejercicio físico de alta intensidad y bajo volumen semanal de entrenamiento, es más eficiente en la reducción de lípidos sanguíneos en comparación a los programas tradicionales de ejercicios aeróbicos.

Lo relevante del presente estudio fue obtener mejoras significativas en estos marcadores sin presentar un diagnóstico previo de hiperlipemia. A diferencia de Álvarez y col. ${ }^{18}$, que trató a pacientes con la enfermedad antes citada, ya que a valores altos y más lejanos a la homeostasis fisiológica son más posibles de disminuir que valores cercanos al rango normal. Esto ha sido señalado por la literatura, tal como lo menciona Blair y col. ${ }^{25}$ y Gill y Malkova $^{26}$. Toledo y Goodspaster ${ }^{27}$, plantean que el mejoramiento en la función oxidativa mitocondrial mediada por ejercicio no necesariamente 
requiere de una modificación sustancial de la composición y peso corporal. La principal limitante de nuestro trabajo fue la pequeña muestra que componía nuestro estudio (33 mujeres), lo que no nos permitió hacer grandes conclusiones y menos generalizar nuestros resultados a la población.

En conclusión, el programa de ejercicio físico de sobrecarga empleado, si bien no modificó el peso ni la composición corporal, logró disminuir los lípidos sanguíneos y el colesterol en el grupo evaluado en comparación a la evaluación inicial. Falta una mayor cantidad de estudios con muestras más grandes y mayor rigurosidad metodológica, pero los resultados clínicos apuntan a que el ejercicio de sobrecarga pudiera ser considerado una buena alternativa terapéutica para normalizar los niveles de lípido y colesterol en sangre y por tanto contrarrestar factores de riesgo cardiovascular en mujeres adultas sedentarias.

Agradecimientos: A los internos de la carrera de Kinesiología de la Universidad Santo Tomás Yanette Plaza y Gabriel Estrada, a la profesora de Educación Física Catalina Cea y a la Universidad de Concepción campus Los Ángeles por su apoyo y colaboración en el estudio.

\section{Referencias}

1. Erikssen G, Liestøl K, Bjørnholt J, Thaulow E, Sandvik L, Erikssen J. Changes in physical fitness and changes in mortality. Lancet 1998; (352): 759-62.

2. Hu FB, Willett WC, Li T, Stampfer MJ, Colditz GA, Manson JE. Adiposity as compared with physical activity in predicting mortality among woman. N Engl J Med 2004; (351): 2694-703.

3. Cigarroa I, Zapata R. Irisina, la hormona que ayuda a quemar grasa. Kinesiología 2013; (32-3): 60-3.

4. Handschin C, Spiegelman BM. The role of exercise and PGC1? in inflammation and chronic disease. Nature 2008; (454): 463-9.

5. Ministerio de Salud Chile. Objetivos sanitarios 20112020: Estrategia nacional de salud para el cumplimiento de los objetivos sanitarios de la década 2011-2020 metas 2011-2020. Santiago, MINSAL (http://web.minsal.cl/ portal/url/item/c4034eddbc96ca6de0400101640159b8. pdf).

6. Haskell W, Lee I, Pate R, Powell K, Blair S, Franklin B, et al. Physical activity and public health: Updated recommendation for adults from the american college of sports medicine and the american heart association. Circulation 2007; 39 (8): 1423-34.

7. Hillman $\mathrm{CH}$, Erickson KI, Kramer AF. Be smart, exercise your heart: exercise effects on brain and cognition. Nat Rev Neurosci 2008; 9 (1): 58-65.

8. Egan B, Zierath J. Exercise Metabolism and the Molecular Regulation of Skeletal Muscle Adaptation. Cell Metabolism 2013; 17 (2): 162-84.

9. Thijssen D, Cable N, Green D. Impact of exercise training on arterial wall thickness in humans Clin Sci (Lond) 2012; 122 (7): 311-22.

10. Booth FW. Waging war on physical inactivity: using modern molecular ammunition against an ancient enemy. J Appl Physiol 2002; (93): 3-30.

11. Zhen Yan M O. Regulation of exercise induced fiber type transformation, mitochondrial biogenesis, and angiogenesis in skeletal muscle. J Appl Physiol 2011; 110: 264-74.

12. Ministerio de Salud Chile. Pontificia Universidad Católica de Chile. Universidad Alberto Hurtado. Encuesta Nacional de Salud 2009-2010. Santiago. (http://web.minsal.cl/portal/url/item/bcb03d7bc28b64dfe040010165012d23.pdf).

13. Instituto Nacional del Deporte (IND). (2012). Encuesta nacional de hábitos de actividad física y deportes en la población chilena de 18 años y más. Facultad de Ciencias Económicas y Administrativas de la Universidad de Concepción, Chile.

14. Gibala M, Little J, Maureen J, Mac Donald M, Hawley J. Physiological adaptations to low-volume, high-intensity interval training in health and disease. J Physiol 2012; 590: 1077-84.

15. Jiménez O, Ramírez R. Strength training improves insulin sensitivity and plasma lipid levels without altering body composition in overweight and obese subjects. Endocrinol Nutr 2011; 58 (4): 169-74.

16. Mancilla R, Torres P, Álvarez C, Schifferli I, Sapunar J, Díaz E. [High intensity interval training improves glycemic control and aerobic capacity in glucose intolerant patients]. Rev Med Chile 2014; 142 (1): 34-9.

17. Jebb S, Cole T, Doman D, Murgatroyd P, Prentice A. Evaluation of the novel Tanita body-fat analyser to measure body composition by comparison with a fourcompartment model. Br J Nutr 2000; (83): 115-22.

18. Álvarez C, Ramírez R, Flores M, Zúñiga C, Celis C. [Effect of sprint interval training and resistance exercise on metabolic markers in overweight women]. Rev Med Chile 2012; 140: 1289-96.

19. Babraj J, Vollaard N, Keast C, Guppy F, Cottrell G, Timmons J. Extremely short duration high intensity interval training substantially improves insulin action in young 
healthy males. BMC Endocrine Disorders 2009; 9-3: 1-8.

20. Paoli A, Moro T, Marcolin G, Neri M, Bianco A, Palma A, et al. High-Intensity Interval Resistance Training (HIRT) influences resting energy expenditure and respiratory ratio in non-dieting individuals. J Trans Med 2012; 237 (10): 1-8.

21. Balducci S, Zanuso S, Nicolucci A, Fernando F, Cavallo S, Cardelli P, et al. Anti-inflammatory effect of exercise training in subjects with type II diabetes and the metabolic syndrome is dependent on exercise modalities and independent of weightloss. Nutr Metab Cardiovasc Dis 2010; 20 (8): 608-17.

22. Haskell $\mathrm{W}$. The influence of exercise training on plasma lipids and lipoproteins in health and disease. Acta Med Scand 1986; (711): 25-33.

23. Boreham C, Kennedy R, Murphy M, Tully M, Wallace W, Young I. Training effects of short bouts of stair climbing on cardiorespiratory fitness, blood lipids, and homocys- teine in sedentary young women. Br J Sports Med 2005; 39: 590-3.

24. Álvarez C, Ramírez R, Flores M, Zúñiga C, Henríquez C, Campos $\mathrm{C}$, et al. [Metabolic response to high intensity exercise training in sedentary hyper-glycemic and hypercholesterolemic women]. Rev Med Chile 2013; 141: 1293-9.

25. Blair SN, Kohl HW, Gordon NF, Paffenbarger RS. How Much Physical Activity is good for Health? Annu Rev Public Health 1992; 13 (1): 99-126.

26. Gill J, Malkova D. Physical activity, fitness and cardiovascular disease risk in adults: interactions with insulin resistance and obesity. Clin Sci (Lond.) 2006; 110 (4): 409-25.

27. Toledo F, Goodpaster B. The role of weight loss and exercise in correcting skeletal muscle mitochondrial abnormalities in obesity, diabetes and aging. Mol Cell Endocrinol 2013; 379 (1-2): 30-4. 